\title{
Coronagraphic Imaging of Debris Disks From a High Altitude Balloon Platform
}

\author{
Stephen Unwin ${ }^{*} a$, Wesley Traub ${ }^{a}$, Geoffrey Bryden ${ }^{a}$, Paul Brugarolas $^{a}$, Pin Chen $^{a}$, \\ Olivier Guyon ${ }^{b}$, Lynne Hillenbrand ${ }^{c}$, John Krist $^{a}$, Bruce Macintosh $^{d}$, \\ Dimitri Mawet ${ }^{e}$, Bertrand Mennesson ${ }^{a}$, Dwight Moody ${ }^{a}$, Lewis C. Roberts Jr. ${ }^{a}$, \\ Karl Stapelfeldt ${ }^{f}$, David Stuchlik ${ }^{g}$, John Trauger ${ }^{a}$, and Gautam Vasisht ${ }^{a}$ \\ ${ }^{a}$ Jet Propulsion Laboratory, California Institute of Technology, Pasadena, CA 91109 \\ ${ }^{b}$ National Observatory of Japan, 650 N. A'ohoku Place, Hilo, HI 96720 \\ ${ }^{c}$ California Institute of Technology, Pasadena, CA 91125 \\ ${ }^{d}$ Lawrence Livermore National Laboratory, Livermore, CA 94550 \\ eEuropean Southern Observatory, Alonso de Córdova 3107, Vitacura, Casilla 19001, \\ Santiago de Chile 19, Chile

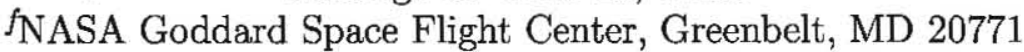 \\ ${ }^{9}$ NASA Wallops Flight Facility, Wallops Island, VA 23337
}

\begin{abstract}
Debris disks around nearby stars are tracers of the planet formation process, and they are a key element of our uncerstanding of the formation and evolution of extrasolar planetary systems. With multi-color images of a significant number of disks, we can probe important questions: can we learr about planetary system evolution; what materials are the disks made of; and can they reveal the presence of planets? Most disks are known to exist only through their infrared fiux excesses as measured by the Spitzer Space Telescope, and through images measured by Herschel. The brightest, most extended disks have been imaged with HST, and a few, such as Fomalhaut, can be observed using ground-based telescopes. But the number of good images is still very small, and there are none of disks with densities as low as the disk associated with the asteroid belt and EdgeworthKuiper belt in our own Solar System.

Direct imaging of disks is a major observational challenge, demanding high angular resolution and extremely high dynamic range close to the parent star. The uitimate experiment requires a space-based platform, but demonstrating much of the needed technology, mitigating the technical risks of a space-based coronagraph, and performing valuable measurements of circumstellar debris disks, can be done from a high-altitude balloon platform. In this paper we present a balioon-borne telescope concept based on the Zodiac II design that could undertake compelling studies of a sample of debris disks.
\end{abstract}

Keywords: Suborbitai, Coronagraph, Debris Disks, Exoplanets

\section{INTRODUCTION}

\subsection{Debris Disks as Signposts of Planets}

Debris disks represent leftover material from the pianet formation process. They are important for study because they provide important insights into the history of the process, and they help us understard our Solar System in the context of the growing body of knowledge of extrasolar planetary systems. Our own Solar System has two belts of these materials, the asteroid belt between Mars and Jupiter, and the Edgeworth-Kuiper belt beyond Neptune. They contain materials with a range of sizes, from micron-sized dust, which would be visible as a debris disk, if viewed from a large distance away, up to planetesimals as large as Pluto. Collisions of the larger

\footnotetext{
*stephen.c.unwin@jpl.nasa.gov

Copyright 2012. All rights reserved.
} 
bociies continually replenish the disk, which is continually depleted by radiation pressure. Sublimation of comets also plays a role.

Our premise in this paper is that we could make significant advances in exoplanetary system science by using a direct-imaging coronagraph, at balloon altitudes, to image debris disks at optical wavelengths. Over the past several years we have studied this concept, here called Zodiac II, and continued to refine its parameters. The current paper is essentially a Design Reference Mission report on Zodiac II.

The smallest-mass particles, micron-sized dust, represent very little mass, but their cross section can cause a dust disk to be apparently brighter than a planet in scattered light. The integrated brightness of the Solar System zodiacal light, at $10^{-7}$ of the Sun, exceeds that of Earth, $10^{-10}$. It is this reflected light that instruments capabie of high-contrast imaging like Zodiac II can detect. Measuring the spatial distribution of the dust emission is important because it may reveal the presence of planets that are too faint to image directly. In our Solar System, Jupiter's gravitational influence prevented the formation of small planets where the asteroid belt is now. Similarly, as Neptune migrated its orbit outward, it pushed material with it, capturing planetisimals into mean-motion resonances, including larger bodies like Pluto (a 3:2 resonance). Direct imaging of the spatial distribution of dust, in rings or bands analogous to structures in the Solar System, especially when combined with color measurements that provide information about grain properties, will allow a better understanding of extrasolar planetary systems, and the history of their formation.

Many disks have been discovered via the dust's thermal emission, since the first image made by IRAS. ${ }^{1}$ The Spitzer Space Telescope has identified disks around many nearby solar-type stars ${ }^{2,3}$ through their excess mid-IR emission with respect to the stellar photosphere. These surveys can only detect emission $\sim 100$ times that of the Solar System's, but they find such bright disks around 15\% of old solar-type stars like the Sun. Some systems have large planets detected via their radial velocity perturbation on the parent star. ${ }^{4}$
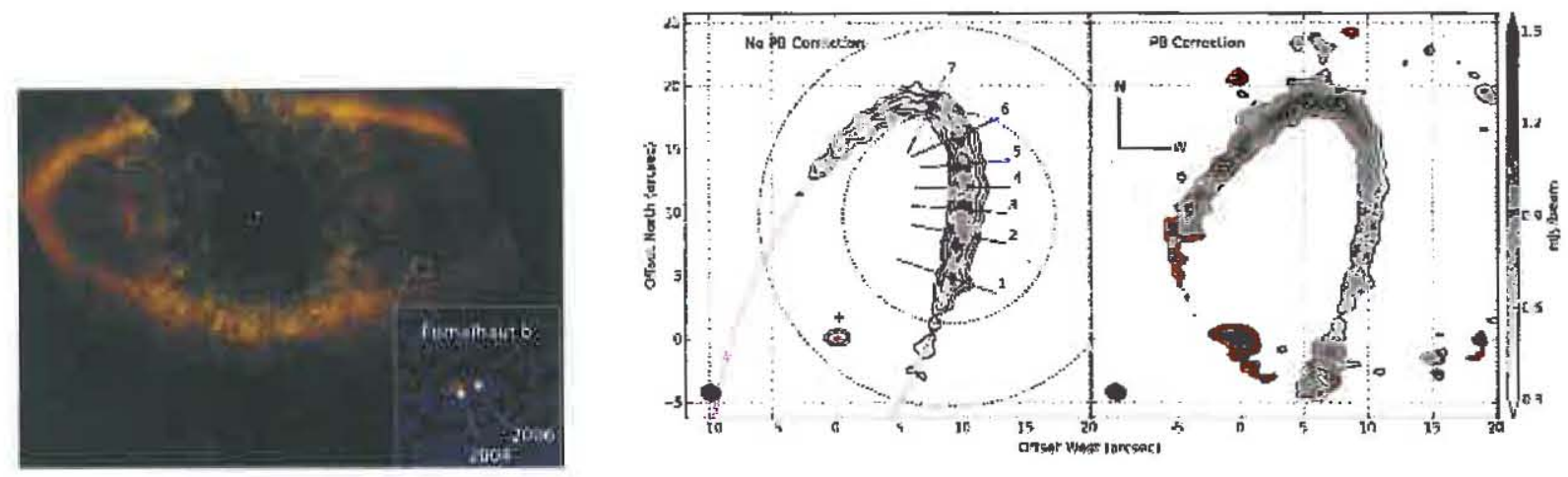

Figure 1. (Left panel) HST image of Fomalhaut's eccentric debris ring and shepherding planet in scattered light. ${ }^{5}$ The dust in this ring orbits at a large separation ( $140 \mathrm{AU}=18 \mathrm{arcsec}$ ). A balloon coronagraph can observe disks that are much fainter and are closer to the central stars (in a radius range $0.3-4$ arcsec). (Right panel) Image of the northern half of the Fomalhaut ring with ALMA at $350 \mathrm{GHz} .^{6}$

The morphology of a debris disk can reveal the presence of hidden planets in many ways - shepherded rings, inclined warps, eccentric offsets, cleared gaps, and resonant clumps. Resolved images of debris disks often reveal asymmetries and warps that may be attributed to the gravitational influence of unseen planets. ${ }^{7-10}$ The best example of a highly resolved debris disk is the remarkably clear dust ring around the A star Fomalhaut. This ring lies on an eccentric orbit that would rapidly disperse in the absence of some shepherding force, presumably a massive planet. ${ }^{11,12}$ The predicted planet has now been directly imaged, using HST (Figure 1 left panel). ${ }^{5}$ However, a sub-mm image made with the new ALMA telescope (Figure 1 right panel), implies the presence of two shepherding planets, neither of which is at the iocation of the planet measured by HST. This model is analogous to the model of Goldreich \& Tremaine ${ }^{13}$ for the role of Cordelia and Ophelia in shaping the $\epsilon$ ring of Uranus. 
HR 8799 has a four-planet system ${ }^{14}$ imaged in L-band (Figure 2) orbiting in the midst of interior and exterior belts of dust; ${ }^{15}$ and Beta Pictoris has an imaged planet that may be responsible for its disk warp. ${ }^{16}$ These are further examples of the strong influence of massive planets on the morphology of debris disk images, and they motivate a desire to obtain resolved images of a much larger sample, using a balloon coronagraph.

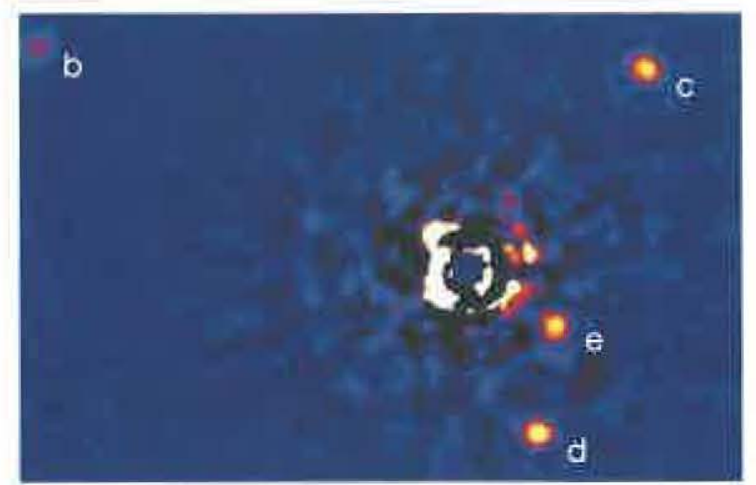

Figure 2. Keck L-band (3.5- $\mu \mathrm{m})$ image of the four planets (b, c, d, \& e) around debris-disk star HR $8799 .{ }^{17}$ A balloon coronagraph such as Zodiac II will be able to images planet $\mathrm{b}$ and $\mathrm{c}$ in visible light for the first time.

\subsection{Measuring Disk Properties}

Observation of these features requires high-resolution imaging at high contrast. Disks are brighter in the thermal IR, but far-IR wavelengths have too low resolution to do more than hint of structure (e.g., Spitzer images of Fomalhaut ${ }^{11}$ ). Optical imaging yields much higher spatial resolution, but in scattered light the contrast relative to the host star is much larger. There are many disks known to exist from their IR excess emission, but even HST cannot image them because the disk contrast is so low. A nigh-contrast coronagraph operating in the optical should be able to image a large number of disks, and this is the objective of the Zodiac II concept.

Five basic properties of debris disks will be revealed by Zodiac II:

Size - the average angular radius of the debris disk, here envisioned as a ring-like structure similar to the Asteroid and Kuiper Belts. Size provides information about the possible location of shepherding planets. The current disk size constrains the size of its original protoplanetary disk.

Shape - three characteristics can be detected by imaging: decentering, waviness, and warps. The disk can be off center with respect to the star if a massive planet is influencing it, particularly one on an eccentric orbit. The disk can have a wave-like edge structure also due to planets. The disk can be warped, with the outer disk misaligned relative to the inner disk, again potentially as a result of planet influence.

Brightness - the surface brightness of a disk, which is directly related to the surface density of material, and also the type of material through its albedio. A combination of infrared and visible flux data can inform us about the temperature and surface density of material, using plausible models of grain size distributions and materials.

Color - the brightness variation as a function of wavelength can tell us about the type of materiai, the size of the individual grains, and their surface reflectivity.

Number - each debris disk is likely to be individual, depending on the particular history of its planetary system. To draw broad conclusions from the observations, statistically significant number of examples is need. Having a number of examples is also important in understanding the life cycle of debris disks as a class. ${ }^{18}$

Making good measurements of these five quantities defines the basic requirements that a balloon coronagraph experiment such as Zodiac II should meet. In particular, given the typical distances of the target stars, the expected brightness of the disks, and the expected physical dimensions, we can directly infer what size telescope is needed, what integration times are needed, and how many targets should be observed.

\subsection{Target Selection}

A balloon-based coronagraph like Zodiac II can potentiaily observe a large list of targets. Details of the selection process are described in Bryden et al. ${ }^{22}$ The starting point is the list of known debris disks within $40 \mathrm{pc}$ of the 

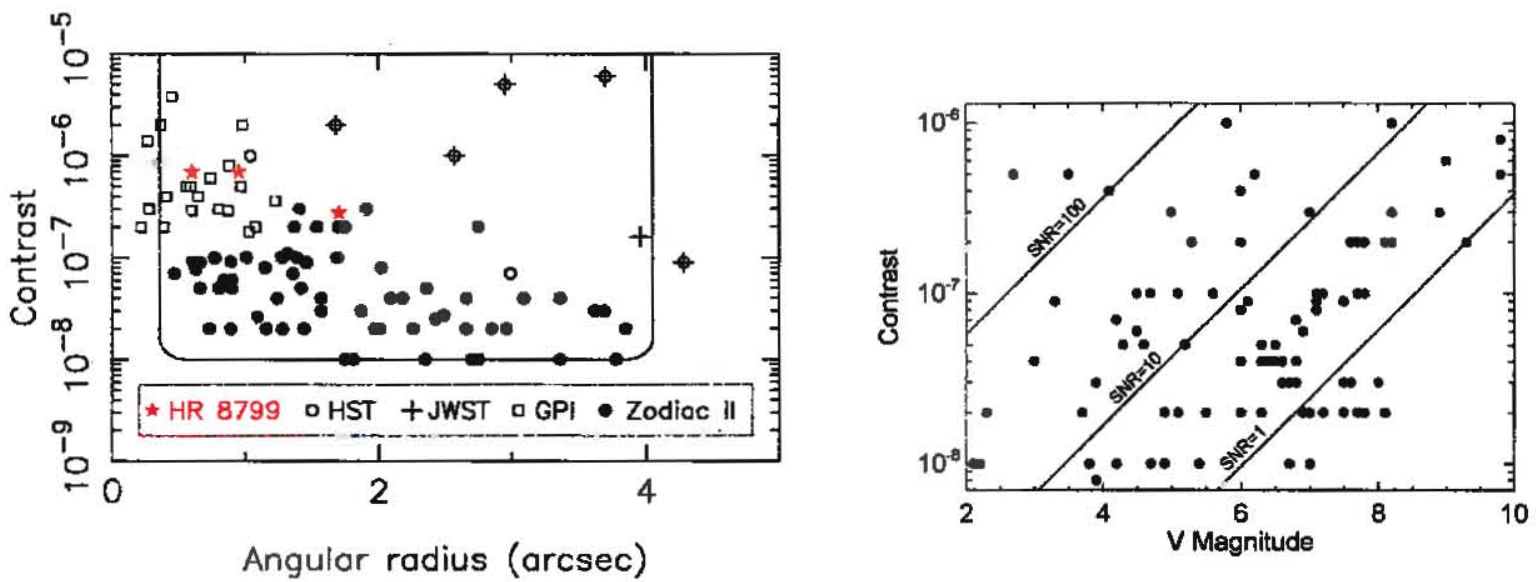

Figure 3. (Left panel) Predicted radii and image contrasts are shown for potential target debris disks, derived from Spitzer data. The inner angular limit is for V1 band $(520 \mathrm{~nm})$; the outer is for I band (900 nm). The 89 filled circles within the U-shaped curve are targets accessible to Zodiac II. Also shown are data points from HST/ACS, ${ }^{19}$ future imaging with Gemini Planet Imager (GPI) ${ }^{20}$ and JWST/NIRCAM in K-band. ${ }^{21}$ (Right panel) SNR achievable by Zodiac II for these targets as a function of stellar magnitude and disk contrast, per resolution element, per hour, in the shortest wavelength (V1) band.

Sun, based on Spitzer detections of their thermal IR emission. ${ }^{2,23-27}$ We include several more identified through WISE and Herschel surveys (e.g., Ref. 28). Although the disks are usually not resolved at infrared wavelengths, we can use their thermal emission to estimate the disk sizes. For estimating the expected signal. levels, we consider uriform surface density rings with width that is $20 \%$ of their radius. The resulting predictions for ring contrasts (with respect to star, per resolution element) are shown in Figure 3. Zodiac II's limits in field-of-view and contrast are shown as a solid U-shaped line, where all points inside the U curve are accessible to Zodiac II in at least one wavelength band. Overall, a large region of the contrast/separation phase space is unique to Zodiac II - at least 89 known debris disks are candidates (filled circles in Figure 3, left panel). For each of these targets, we caiculate the expected debris disk signal-to-noise ratio (SNR) based on a 1-hour observation (Figure 3, right panel). For simple detection of the existence of a ring, post-processing will increase these values by combining data from all points along the disk and over all 4 wavebands for a net increase in SNR by a factor of about 20 .

While debris disks are the primary focus of Zodiac II, the brightest known exoplanets can also be targeted. Several coronagraphs with advanced adaptive optics systems are now coming online (e.g. GPI, P1640, and VLT/SPHERE), ${ }^{20,29,30}$ so additional targets for optical imaging are expected to emerge in the next few years. Two of the four known exoplanets in the HR 8799 system (Figure 2) are detectable; SNR for a 1 hour integration are about 50 and 90 for for HR $8799 \mathrm{~b}$ and c respectively ${ }^{14,17}$ (the inner two planets, $\mathrm{d}$ and e, fall inside the I-band inner working angle and are too faint for observations in $\mathrm{V}$ band). Optical detection would be very significant: atmosphere models predict these objects should be $\sim 5$ mag fainter in I band than in $\mathrm{K}$, due to their low effective temperatures and strong pressure-broadened absorption of neutral potassium, ${ }^{31}$ but these models have never been observationally tested.

\section{CORONAGRAPH EXPERIMENT}

We present a balloon telescope experiment based on the proposed Zodiac II design, which would undertake compelling studies of a sample of debris disks while at the same time advancing key technologies. The system is described in detail by Bryden et al., ${ }^{22}$ and we summarize the key features here. We are considering two changes in the system for future development: (1) the use of a lightweighted glass primary mirror (though the SiC mirror selected for Zodiac II has many important advantages); (2) the ability for the system to accept multiple backend coronagraphs. The baseline is the most mature type, the band-limited Lyot coronagraph, but there is 
considerable interested in flying alternate designs, including a vector vortex coronagraph; ${ }^{32}$ the current concept is to switch backends between flights (see Section 2.3). There has also been significant progress in the precision telescope pointing system, important for all coronagraphs, and we describe results from a successful flight in Section 2.2 below.

A high-altitude balloon can carry a telescope payload to a near-space environment, which high-contrast imaging experiments are possible, above almost all of the disturbing effects of the Earth's atmosphere. Besides cost, balloons have a major advantage over space-based platforms: the payload can be recovered after flight, modified or refurbished, and re-flown. Experience shows that platforms that are designed to minimize damage upon landing do in fact allow instrument re-use. We propose to take advantage of this unique feature of a balloon platform to allow testing of different coronagraph architectures. A number of different coronagraph designs have been tested in a laboratory environment, and some have been implemented in ground-based imaging of the brightest extended debris disks, such as Fomalhaut ${ }^{6,8}$ and HR $8799 .^{33}$ The next logical step toward a space mission is a balloon-borne experiment that takes coronagraph performance to the next level. Image contrast at a ground telescope is fundamentally limited by the atmosphere, so a coronagraph cannot be tested at the contrast ievel appropriate for a space mission. However, at the altitude of a high-altitude scientific balloon $(\sim 35 \mathrm{~km})$, the atmospheric seeing contribution to high-contrast imaging is very small (Section 2.3). The system would have a modular backend design, to accommodate different coronagraph architectures, ailowing us to gain experience with different instruments on subsequent flights of the telescope payload.

Critical to the performance of a coronagraph in air is the effect of seeing on image contrast. The best groundbased telescopes reduce seeing speckles with adaptive optics (AO) to reduce speckles, with a practical limit in the visible of $\sim 10^{-7}$ of the star for an optimized instrument such as VLT-SPHERE. Calculations by Bryden et al. ${ }^{22}$ show that at the Zodiac II altitude, beyond $0.2 \mathrm{arcsec}$ from the central star the expected speckles are no larger than $10^{-10}$ and falling rapidly with radius.

\subsection{Gondola and telescope}

The gondola and telescope body pointing system are provided by the NASA Wallops. Flight Facility (WFF) based on their experience with balloon payloads and in particular their previous development of the Wallops Arc Second Pointer (WASP) pointing system. ${ }^{34}$ The gondola hangs from a coarse azimuth-driver at the end of the balloon train. The telescope frame is open to allow air to flow unimpeded across the optical path, to minimize locally-generated turbulence.

The telescope for Zodiac II has four key characteristics. (1) Light weight - silicon carbide (SiC) or lightweighted glass are attractive for getting a payload to $35 \mathrm{~km}$ altitude with a moderate size balloon. For example, the total mass of a SiC-primary telescope would be around $200 \mathrm{~kg}$. (2) Unobstructed aperture - the coronagraph performance is severely compromised if the telescope has a central obscuration, so an off-axis design is essential. (A visible nulling coronagraph would be able to work around this limitation, but the choice of instrument involves many other considerations, see below). (3) Large diameter - bigger is better, both for sensitivity (minimize observing time on target), and for angular resolution for spatially resolving the target debris disks. (4) Optical quality - the svstem must deliver to the coronagraph a wavefront corrected for tip/tilt and with focus/astigmatism errors no larger than $18 \mathrm{~nm}$ RMS; and mid-spatial frequency mirror surface errors (2-28 cycles/m) no larger than $3 \mathrm{~nm}$ RMS. Note that this greatly exceeds the requirements for a telescope that delivers normal diffraction-limited images.

The thermal design of the system is important for maintaining image quality. Rather than trying to let the telescope equilibrate at altitude (time constant $\sim 30$ hours for a $\mathrm{SiC}$ mirror), we keep the primary mirror and instrument package close to room temperature at all times. Local 'seeing' about the primary mirror is a consideration, but we can show that turbulence is negligible (Grashof and Reynolds numbers well below the critical values). The only effect expected is a slowly varying 'wedge' that can be taken out by a low-order deformable mirror (see below).

\subsection{Pointing System}

All internal coronagraphs for high-contrast imaging have stringent requirements for pointing stability and control. Pointing offsets adversely affect image contrast in two main ways: 1) jitter and stability errors in the telescope 
line-of-sight pointing lead to contrast degradation due to wavefront errors generated by beam-walk, and 2) errors in centering the occulting mask accurately on the target star produce contrast degradation cue to light spillage outside the mask. Ref. 35 calculated the contrast degradation sensitivities due to errors in telescope pointing and mask centering (see also Refs. 36-38). The pointing stability requirements for Zodiac II are guided by those calculations, which translate to a. 0.4 arcsec RMS line-of-sight stability for the telescope body-pointing and a 0.04 arcsec RMS coronagraph mask centering error. Telescope roll around the line-of-sight is not actively controlled. The roll motions due to field rotation $(<0.3 \mathrm{deg} / \mathrm{min})$ and non-controlled gondola roll $\left(<0.5^{\circ}\right)^{39}$ are small compared to the roll pointing requirement of $1.2^{\circ}$ over $60 \mathrm{~s}$ and do not affect the performance of the instrument.

A key component of the Zodiac II system is the telescope pointing system. We use the WASP ${ }^{34}$ system, which eliminates static friction by keeping an inner race of the bearing constantly rotating at a slow speed. WASP bearings are used in both altitude (elevation) and azimuth (or cross-elevation).

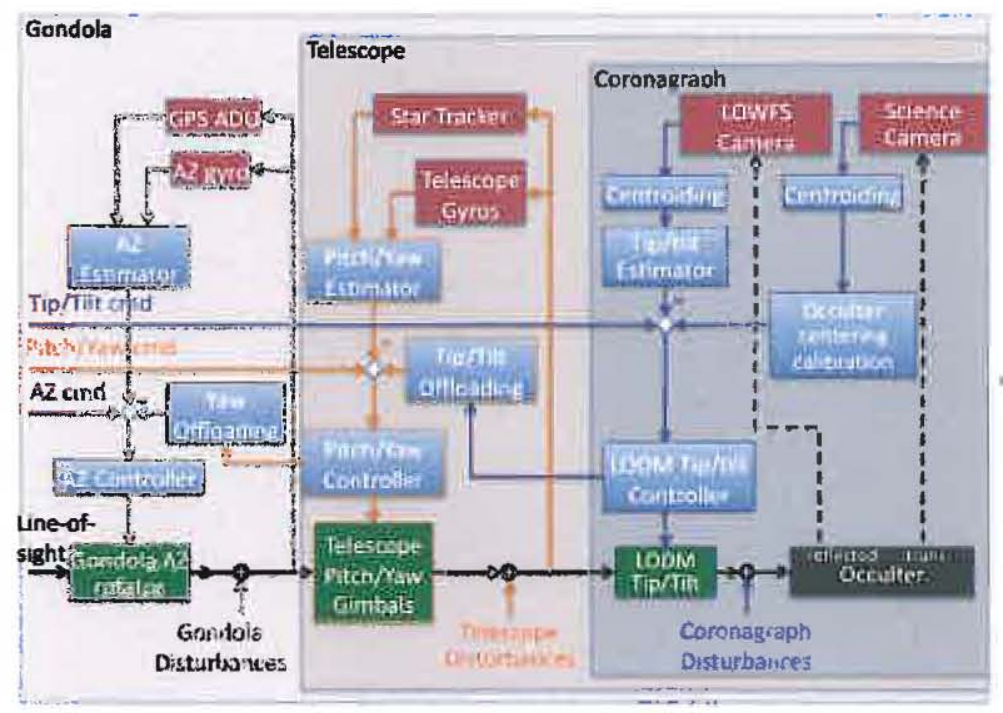

Figure 4. Pointing control flow diagram. The three stages of control utilize the gondola azimuthal rotator (outer pinkshaded box), telescope pitch and yaw gimbals (middie orange-shaded box) and coronagraph internal tip/tilt (inner blueshaded box). Actuators are depicted in green, sensors in red, algorithms in blue, and the coronagraph occulter in grey.

To meet its pointing requirements Zodiac II lises a 3-stage pointing system. Figure 4 shows a control system diagram for this nested 3-stage pointing architecture. First, the gondola azimuth pointing stage provides coarse pointing of the gondola and telescope in azimuth. A GPS attitude determination unit and a single axis gyro are used to estimate the azimuth direction of the gondola; the gondoia is then pointed in the desired azimuth direction. This first stage has low bandwidth $(<0.1 \mathrm{~Hz})$. Second; the telescope gimbal system provides telescope pitch and yaw pointing. The telescope pointing svstem estimates the attitude and attitude rate of the telescope by filtering the star tracker and gyro measurements. Then it servos the pitch and yaw gimbals to point the telescope to the target star. The gimbal servo system runs at medium bandwidths $(<10 \mathrm{~Hz})$. For the third pointing stage, the finest scale adjustments are made by a tip-tilt system inside the coronagraph instrument which centers and stabilizes the occulting mask on the target star. The integral tip/tilt system has \pm 60 arcsec of tip/tilt motion, which translates to a line-of-sight range of motion projected onto the sky of \pm 5.2 arcsec. The sensor driving the tip-tilt is a 16x16 Shack-Hartmann wavefront sensor operating at a $500 \mathrm{~Hz}$ frame rate. Each subaperture is $4 \times 4$ pixels plus a 1 pixel guard band. The wavefront sensor estimates the position of the central star to 0.01 arcsec or better. This level of performance is due to the fact that the selected central stars are bright and the telescope has a large aperture. The combination provides ample signal for the wavefront sensor to support the high gain-bandwidth tip/tilt loop $(<100 \mathrm{~Hz})$. 

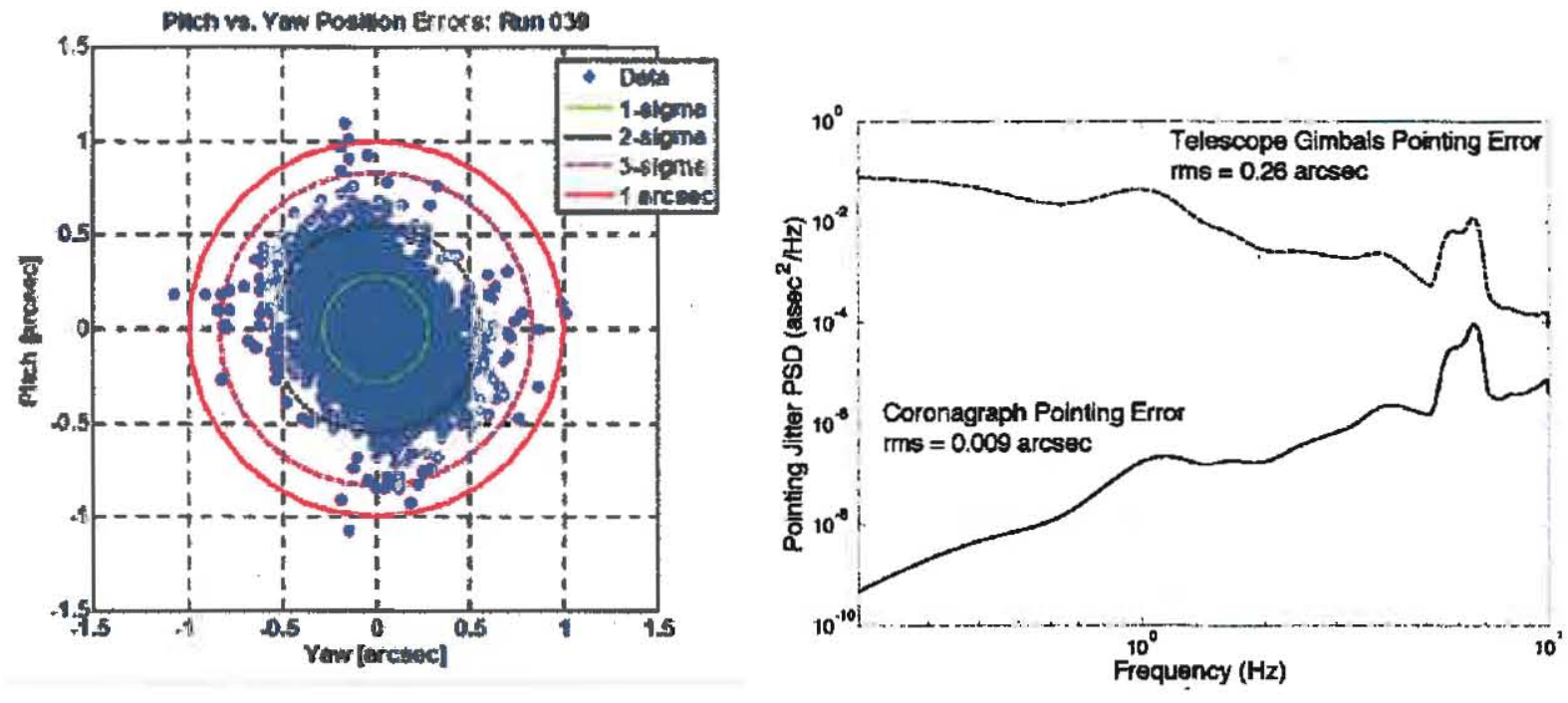

Figure 5. (Left panel) Results from a laboratory experiment measuring residual tip/tilt jitter of the telescope platform after the gimbal control loops are closed (courtesy K. DeWeese ${ }^{34}$ ). The residuai jitter is 0.25 arcsec RMS, satisfying Zodiac II's 0.4 arcsec RMS requirement. (Right panel) Measured WASP telescope gimbals pointing error (dashed) and estimated compensated coronagraph pointing error (solid) with the tip-tilt pointing correction.

The main sources of disturbances that need to be compensated for are balloon pendulations and gondola bearings disturbances. Balloon pendulations gererate low amplitude $\left(<1^{\circ}\right)$ low frequency $(<1 \mathrm{~Hz})$ pointing errors. $^{39}$ The fine steering mechanism could act as a disturbance by exciting the structure and inducing pointing errors. However, since the range of motion is small, and the mass participation of the moving parts is also small, the induced disturbance is negligible. The telescope/gondola pointing system has been demonstrated in the laboratory, ${ }^{34}$ with the pitch and yaw gimbal system meeting the 0.4 arcsec pointing requirement (Figure 5 , left panel).

A successful flight test of the WASP was conducted in October 2011. Photos of the preparations for this flight test are shown in Figure 6. For this experiment, the telescope structure was simulated by a massive beam to serve as a reaction mass. Results from the experiment are shown in Figure 7. While the system is tracking the RMS pointing jitter is 0.23 arcsec, and residual oscillations are simple pendulum mode, with 20-25s period.

To assess the coronagraph fine pointing capability needed for centering a star on the coronagraph mask, and to evaluate the pointing stability of the telescope pointing gimbals in conjunction with the coronagraph's tip-tilt fine steering, we developed a preliminary design for the fine steering control loop and analyzed its performance against data from a realistic control closed-loop experiment of a prototype of the Wallops gimbals system. ${ }^{34}$ The right panel of Figure 5 shows the uncompensated pointing jitter residual from a telescope gimbal (dashed) and the tip/tilt compensated pointing jitter (solid). As a result of this third pointing stage, the pointing error is reduced from 0.26 arcsec RMS to 0.009 arcsec RMS.

\subsection{Coronagraph}

Direct imaging of debris disks and debris disks requires very high contrast imaging. This is the primary motivation for an experiment above the disturbing effects of the atmosphere. A coronagraph is the essential feature of the backend instrument, blocking the light of the central star, and allowing the imaging system to record the much fainter emission from the circumstellar material. There are a number of distinct designs for coronagraphs. For an extended discussion of direct imaging by coronagraphs, see the book chapter by Traub \& Oppenheimer in ref. 41. 

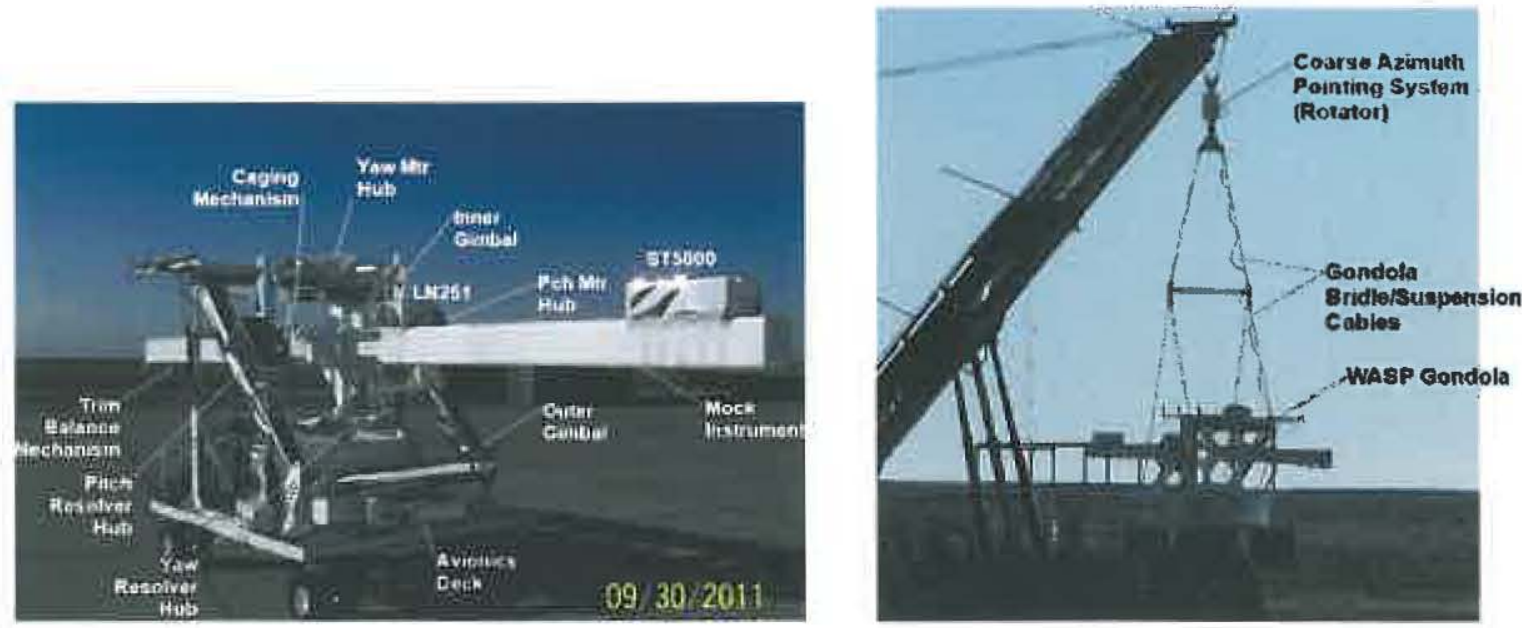

Figure 6. Photos of the preparations for flight test of the Wallops Arc Second Pointer (WASP), October 2011. ${ }^{40}$
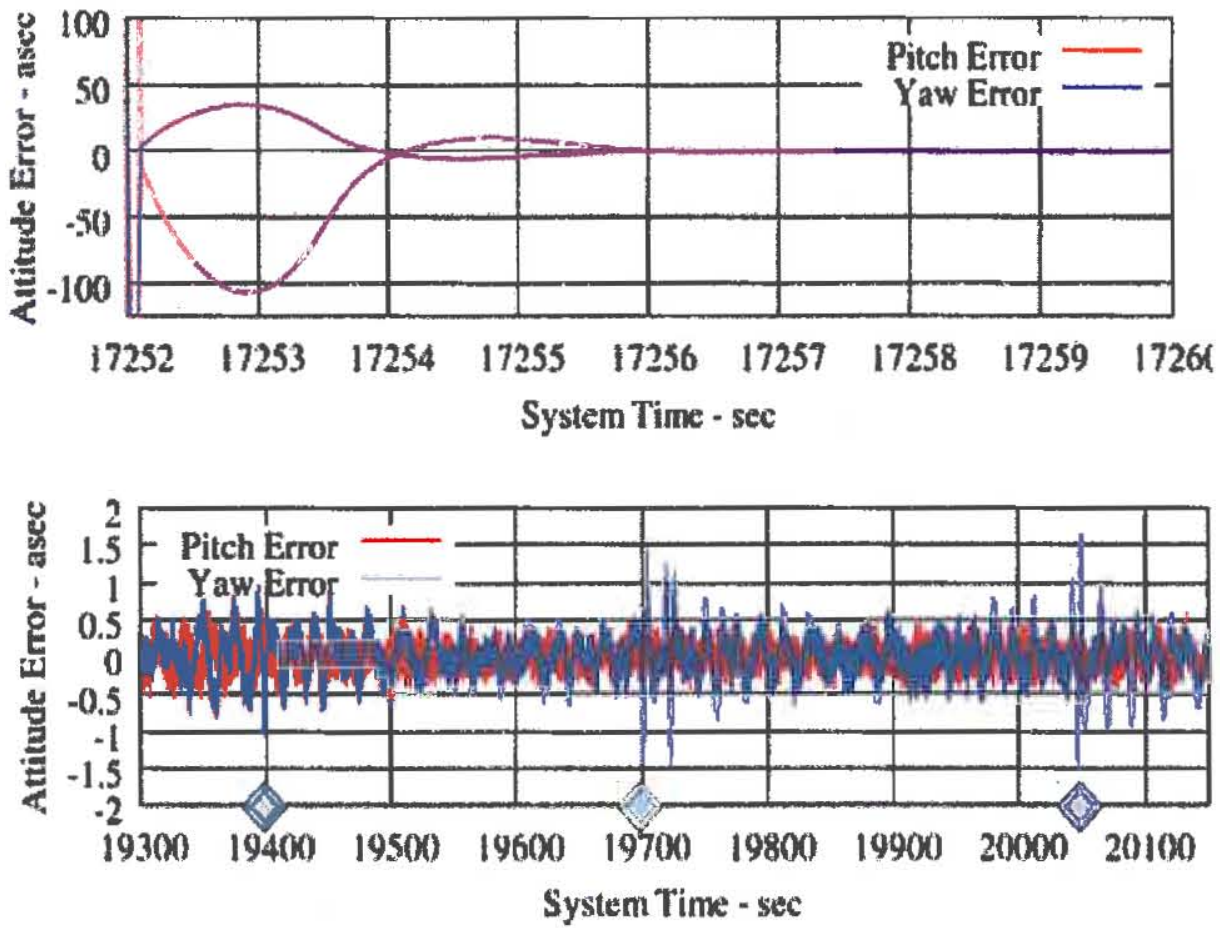

Figure 7. Results from a flight test of the WASP, September 2011. ${ }^{40}$ Payload operated for two hours at an altitude of $105,000 \mathrm{ft}$. (Top) Response of the system to a large slew, showing settling completed less than $4 \mathrm{~s}$ after completion of the slew. (Bottom) Pointing performance after three rotor changes (diamond markers) is 0.23 arcsec RMS. 


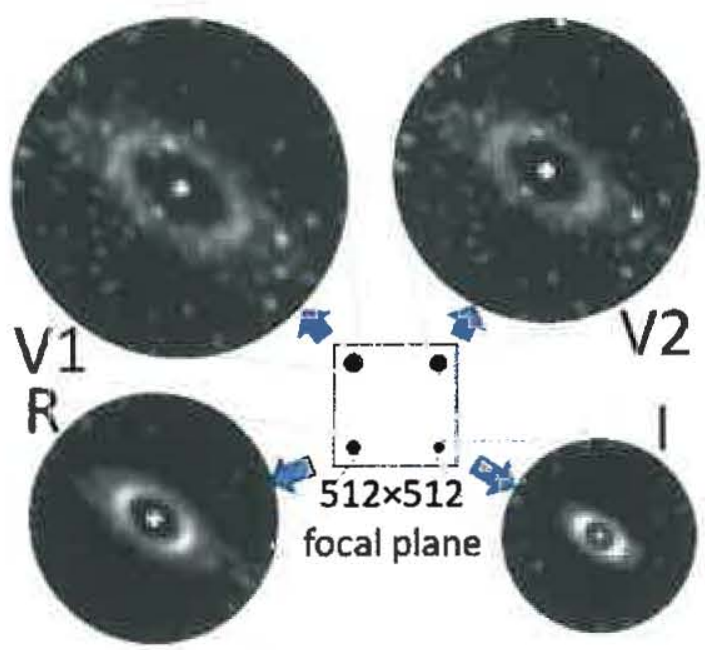

Figure 8. Simulation of the raw (unprocessed) imaging performance of Zodiac II. Enlarged images for each of the four wavelength bands are shown in the corners, while their positions on the 512x512 pixel CCD camera are marked in the central square, to scale. A disk structure with two belts at different temperatures is assumed to surround a $V=6$ star. After 1 hour of 1-minute integrations, the inclined disk within each of the circular dark holes is seen to stand out clearly against the background of semi-random speckles. Residuals from the wavefront correction are apparent, but post-processing software further reduces these speckles. The optics ensure that each image has sub-Nyquist sampling (2.5 pixels per FWHM), making the longer wavelength R- and I-band images smaller on the CCD than the V-band images.

Zodiac II baselines the hybrid band-limited Lyot coronagraph (HBLC), based on an extremely high performance design achieving contrasts in a stable lab environment at a level of sensitivity a factor of 1000 better than needed for Zodiac II. ${ }^{42}$ The heart of the Zodiac II coronagraph is a focal-plane band-limited mask that suppresses the telescope's diffraction pattern. Diffracted light is blocked by putting a precisely tapered dark spot in the focal plane that is $\sim 3$ times larger than the core size of the star $\left(\lambda / D_{t e l}\right.$ in angle on the sky) and surrounding the spot with a pattern of faintly darkened lines or circles. The central dark spot eliminates the main part of the starlight, and the faint pattern eliminates all the diffracted Airy rings.

The incident wavefront must be nearly perfect for the mask to operate correctly; so two deformable mirrors (DMI) precede it: a low-order deformable mirror (LODM) mounted on a tip-tilt stage followed by a high-order DM (HODM) for even finer wavefront corrections. A simulated image of a debris disk, with superposed speckles, is shown in Figure 8.

Telescope imperfections cause speckles that create a noise floor for any observations. This floor is not constant, but is composed of many slowly varying point-source-like speckles. While for conventionai imaging these errors would be barely discernible, for coronagraphy, they must be corrected. This is done by inserting the DMs in the beam of light, between the telescope and the focus. The resulting 'dark hole' is the high-contrast region where one can image debris disks and exoplanets.

Figure 9 shows a schematic of the coronagraph optics layout for Zodiac II. The coronagraph is a flight version of tie High-Contrast Imaging Testbed (HCIT) which has been in operation in the lab at JPL for over 6 years and has demonstrated contrast ratios of $6 \times 10^{-10}$ with a stability of $0.1 \times 10^{-10}$ RMS over 5 hours. ${ }^{42}$ This substantially exceeds the performance needed for a balloon coronagraph.

Critical elements of the design are the wavefront sensing and control subsystems. Two deformable mirrors are used to correct the wavefront. The LODM corrects low-order aberrations such as tip-tilt, focus and astigmatism, through the first 15 Zernike modes, using a 469 channel Xinetics DMI mounted on a tip-tilt stage. ${ }^{43}$ High spatial frequency errors are corrected with a second stage - the HODM - which is a Xinetics $48 \times 48$ deformable mirror with a continuous face sheet. The low-order wavefront sensor (LOWFS) measures the low-order aberrations using a Shack-Hartmann sensor, and these data are used to drive the LODM and its associated tip-tilt stage. It 


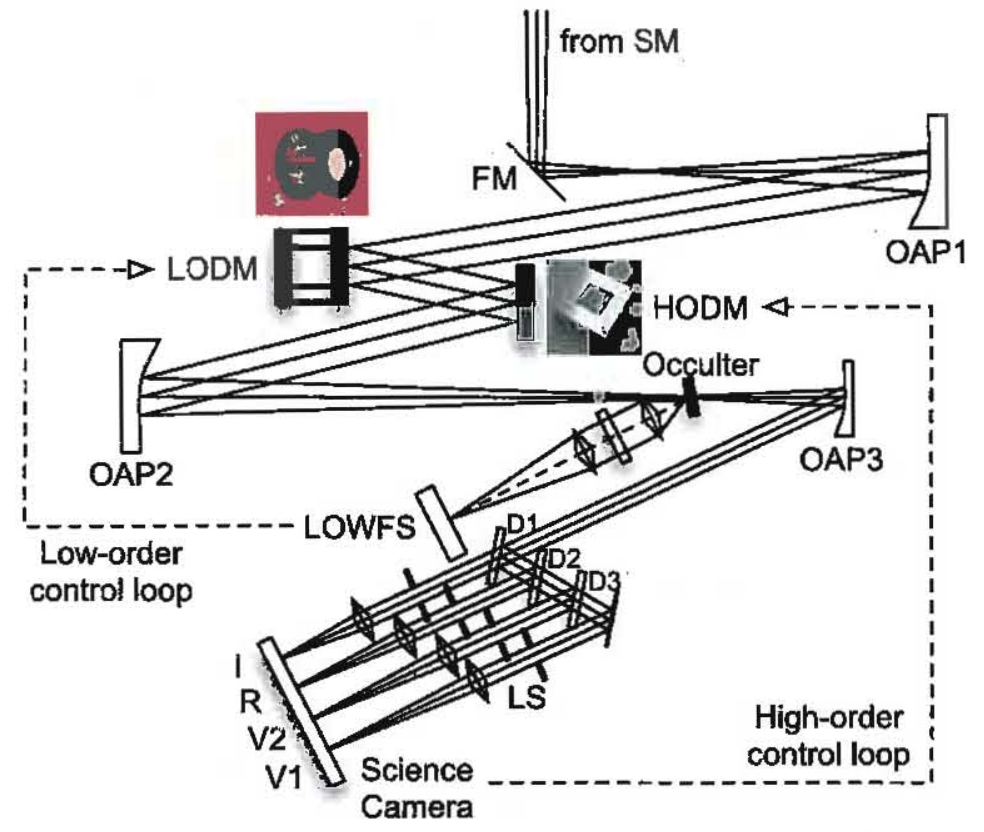

Figure 9. Coronagraph schematic (not to scale) showing optical path (solid lines), feedbacks (dashed lines), and photos of the existing LODM and HODM components. The low-order modes are measured by a LOWFS using light reflected off the occulter. These modes are corrected by the LODM. The high order deformable mirror is adjusted based on science camera images of the residual speckles. Dichroics (D1, D2, D3) split the beam into 4 wavelength bands that pass through individual Lvot stops (LS) before reaching the single science camera.

receives the light reflected from the front of the coronagraph mask. The LOWFS detector is an $80 \times 80$ frametransfer CCD that is widely used as the wavefront sensor for ground-based adaptive optics, such as the Palomar Adaptive Optics system. ${ }^{44}$ The LOWFS includes a field stop, collimating lens, and lenslet array.

Two wavefront sensing and control methods are used to adjust the deformable mirrors in Zodiac II. Each avoids non-common-path errors by using only imagery of a star at the science focal plane. The Gerchberg-Saxton method $^{45}$ is used for initial telescope alignment and DM settings, based on analysis of defocused images of a target star with the coronagraph elements temporarily removed from the beam. The Electric Field Conjugation (EFC) method, ${ }^{46,47}$ which probes the wavefront amplitude and phase with four discrete settings of the DMs, is appiied iteratively to suppress scattered light within the coronagraph dark field. Typical convergence rates for the EFC method are illustrated in Figure 10 which shows calibration times to reach a range of contrast levels for laboratory data (red) and corresponding simulations (blue). The speed of convergence of WFE control actions is determined by the count rate of electrons in each detector. The figure shows a family of convergence curves based on our theoretical SNR for stars of magnitude $V=0$ to 8 , and in particular shows that for a bright star the convergence time to achieve a speckle contrast of $10^{-7}$ is in the range 12-30 minutes. The connected circles show the actual convergence times on the HCIT, where the same general trend of convergence is seen, validating our expectations for Zodiac II.

The science camera records the images from all four wavebands in parallel, with each band falling in a separate quadrant of the array (see Figure 8). The camera also provides the information for real-time speckle suppression by the deformable mirrors. The camera detector is an e2v CCD57-10, a frame-transfer device enabling a lowspeed read out, minimizing read noise, and eliminating the need for a shutter. It is thermoelectrically cooled to $-35^{\circ} \mathrm{C}$ to reduce dark current. We baseline 60-s integrations with this detector, to balance read noise and dark count noise and to minimize snapshot time compared to the time scale of potential wavefront changes.

\section{FLIGHT OPERATIONS DATA ANALYSIS}

Single-night test flights from Ft. Sumner, NM will be scheduled during the turnaround times when stratospheric winds are weakest (late spring and early fall), minimizing the amount of drift during the 24-hour flights. Depending on initial flight experience, we would switch out the coronagraph between flights, to gain experience in 


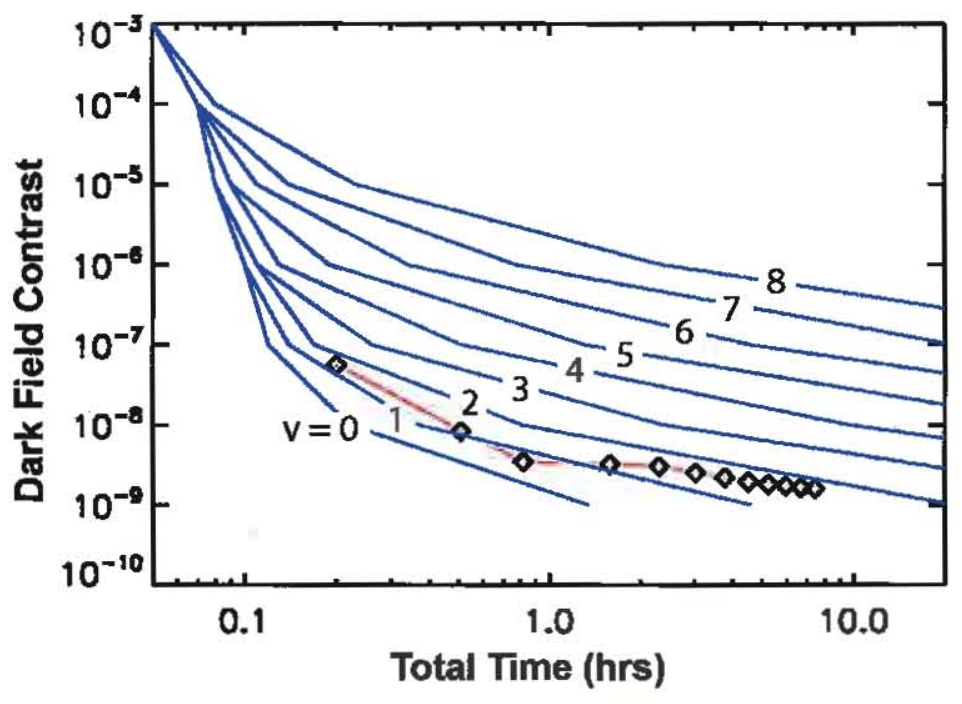

Figure 10. Contrast achieved in the coronagraph dark hole as a function of time. Results from the High-Contrast Imaging Testbed (HCIT), using EFC, are shown as a red line. Based on our SNR model for Zodiac II, we calculated dark hole convergence rates for targets of varying brightness (blues lines for $\mathrm{V}=0$ to $\mathrm{V}=8$ mag stars). For bright calibrator stars, the dark hole reaches a contrast of $10^{-7}$ in tens of minutes. The theoretical curves are validated by the HCIT lab data.

operating these instruments in a near-space environment. Each overnight flight will have about $10 \mathrm{hr}$ observing time, which will be devoted to approximately 4 targets.

Science and engineering data are continually stored on board, with a subset sent to ground via telemetry. All four wavelength channels are imaged onto a single detector. The four dark holes together contain about 34,000 pixels. During the observation of a target, consecutive exposures of approximately one minute each will be taiken. The full CCD frame will be stored onboard ( $\simeq 30 \mathrm{MB}$ per hour) for retrieval after payload recovery.

All of the control subsystems such as the various pointing mechanisms (telescope pointing, tip-tilt mirror), the derived wavefront measurements, and the DM state, will generate data that can be used a posteriori to retrieve the state of the system at any given time. This information can be exploited to enhance the data reduction process. The engineering data rate is roughly a quarter that of the science rate. All data will be stored onboard and retrieved after recovery of the payload.

After each flight, advanced image processing will be used to remove residual speckles and thereby reveal faint circumstellar structure. As seen in the simulated images in Figure 8, which includie wavefront propagation through the coronagraph and correction cycles, residual scattered starlight forms a field of speckles with a surface brightness that can be comparable to that of a disk. Speckles, which appear static on the detector because they are created by the optics, can be subtracted using an iterative algorithm that solves for the instrumental effects (static features) and the sky (features that rotate with the parallactic angle). This algorithm has been successfully used on HST data to extract images of debris disks (beta Pic ${ }^{48}$ and HD $207129^{49}$ ). The same algorithm was used to extract simulated planets from actuai HCIT coronagraphic images. ${ }^{42}$

Zodiac II's wavelength coverage in 4 bands provides additional information for differentiating between instrumental speckles and real sky sources. HCIT lab results implv that at the level of contrast probed by Zodiac II $\left(\sim 10^{-7}\right)$, the wavefront errors will be mostly phase perturbations, so resulting speckle positions will then be highly correlated with wavelength, allowing us to take use the 4 wavebands to remove them. All removal methods rely on the time stability of the speckles, and hence the wavefront, as any changes would introduce residuals in the disk image. A factor of 10 reduction in background should be easily achieved, based on recent experience with space and ground-based coronagrahy. ${ }^{19,29,33,50}$

\section{CONCLUSIONS}

The quest to understand the nature of extrasolar planets and their formation and evolution has developed in recent years into a very rich area of research. It is a fundamental goal of astrophysics that will continue so 
drive science and technology development. Zodiac II represents an important incremental step along that path - both in technology development and in science. Before a full flagship mission for direct imaging of Earthlike planets can be developed, reconnaissance surveys must be carried out to help set the requirements. Both planet and debris disk observations are necessarv. Debris disks represent important science targets in their own right (Section 1), but for exoplanet imaging, they represent a source of noise. And if they contain significant substructure, they may be a source of confusion as well, as shown by Roberge. ${ }^{51}$ We know that debris disks are common, from the IR excesses measured by Spitzer, but a measure of the total amount of dust is not sufficient we need to measure and understand the distribution of the deleterious dust. The Zodiac II observatory is capable of resolving disks in 4 optical colors. These images will contain enough information to measure the size, shape, brightness, and color of a statistically significant number of debris disks. This will inform us about the general evolution of planetary disks and give clues to the specific locations where individual planets may be found.

Zodiac II is also a technology pathfinder, providing a test environment representative of space for a tiny fraction of the cost, and much faster, than a major space observatory. We already have extensive laboratory experience in high contrast imaging, through testbeds like the HCIT. ${ }^{42} \mathrm{~A}$ bailoon platform such as Zodiac II represents an important bridge to space, in terms of demonstratirg the technology that a large space-based coronagraph will need for imaging of potentially-habitable worlds around nearby stars.

\section{ACKNOWLEDGMENTS}

Some of the research described in this publication was carried out at the Jet Propulsion Laboratory, California Institute of Technology, under a contract with the National Aeronautics and Space Administration.

\section{REFERENCES}

1. Aumann, H. H., "IRAS observations of matter around nearby stars," PASP 97, 885 (1985).

2. Trilling, D., Bryden, G., Beichman, C., et al. "Debris Disks around Sun-like Stars," ApJ 674, 1086 (2008).

3. Lawler, S. M., Beichman, C. A., Bryden, G., et al., "Explorations Beyond the Snow Line: Spitzer/IRS Spectra of Debris Disks Around Solar-Type Stars," ApJ 705, 89 (2009).

4. Bryden, G., Beichman, C. A., Carpenter, J. M., et al., "Planets and Debris Disks: Results from a Spitzer/MIPS Search for Infrared Excess," ApJ 705, 1226 (2009).

5. Kalas, P., Graham, J. R., Chiang, E., et al., "Optical Images of an Exosolar Planet 25 Light-Years from Earth," Science 322, 1345 (2008).

6. Boley, A. C. and Payne, M. J. and Corder, S. and Dent, W. R. F. and Ford, E. B. and Shabram, M. "Constraining the Planetary System of Fomalhaut Using High-Resolution ALMA Observations," ApJL 750, L21 (2012).

7. Wyatt, M. C., Dermott, S. F., Telesco, C. M., et al. "How Observations of Circumstellar Disk Asymmetries Can Reveal Hidden Planets: Pericenter Glow and Application to the HR 4796 Disk," ApJ 527, 918 (1999).

8. Kalas, P., Graham, J. R., Clampin, M. C., and Fitzgerald, M. P., "First Scattered Light Images of Debris Disks around HD 53143 and HD 139664," ApJL 637, L57 (2006).

9. Chen, C. H., Sheehan, P., Watson, D. M., et al., "Solar System Analogs Around IRAS-Discovered Debris Disks," ApJ 701, 1367 (2009).

10. Chiang, E., Kite, E., Kalas, P., Graham, J. R., and Clampin, M., "Fomalhaut's Debris Disk and Planet: Constraining the Mass of Fomalhaut b from disk Morphology," ApJ 693, 734 (2009).

11. Stapelfeldt, K. R., Holmes, E. K., Chen, C., et al., "First Look at the Fomalhaut Debris Disk with the Spitzer Space Telescope," ApJS 154, 458 (2004).

12. Kalas, P., Graham, J. R., and Clampin, M., "A planetary system as the origin of structure in Fomalhaut's dust belt," Nature 435, 1067 (2005).

13. Goldreich, P., \& Tremaine, S. Nature 277, 97 (1979).

14. Marois, C., Macintosh, B., Barman, T., et al., "Direct Imaging of Multiple Planets Orbiting the Star HR 8799," Science 322, 1348 (2008).

15. Su, K. Y. L., Rieke, G. H., Stapelfeldt, K., et al. "The Debris Disk Around HR 8799," ApJ 705, 314 (2009). 
16. Lagrange, A., Bonnefoy, M., Chaurin, G., et al., "A Giant Planet Imaged in the Disk of the Young Star $\beta$ Pictoris," Science 329, 57 (2010).

17. Marois, C., Zuckerman, B., Konopacky, Q. M., et al., "Images of a fourth planet orbiting HR 8799," Nature 468, 1080 (2010).

18. Wyatt, M. C., "Evolution of Debris Disks," ARAA 46, 339 (2008).

19. Krist, J. E., "High-contrast imaging with the Hubble Space Telescope: performance and lessons learned," Proc. SPIE 5487, 1284 (2004).

20. Macintosh, B. A., Graham, J. R., Palmer, D. W., et al., "The Gemini Planet Imager: from science to design to construction," Proc. SPIE 7015 (2008).

21. Beichman, C. A., Krist, J., Trauger, J. T., et al., "Imaging Young Giant Planets From Ground and Space," PASP 122, 162 (2010).

22. Bryden, G., Traub, W., Roberts, Jr., L. C., Bruno, R., Unwin, S., Backovsky, S., Brugarolas, P., Chakrabarti, S., Chen, P., Hillenbrand, L., Krist, J., Lillie, C., Macintosh, B., Mawet, D., Mennesson, B., Moody, D., Rahman, Z., Rey, J., Stapelfeldt, K.; Stuchlik, D., Trauger, J., Vasisht, G., "Zodiac II: debris disk science from a balloon',' Proc. SPIE 8151, 81511E (2011).

23. Su, K. Y. L., Rieke, G., Stansberry, J., et al. "Debris Disk Evolution around A Stars," ApJ 653, 675 (2006).

24. Hillenbrand, L. A., Carpenter, J. M., Kim, J. S., et al., "The Complete Census of $70 \mu \mathrm{m}$-bright Debris Disks within 'the Formation and Evolution of Planetary Systems' Spitzer Legacy Survey of Sun-like Stars," ApJ 677, 630 (2008).

25. Bryden, G., Beichman, C. A., Carpenter, J. M., et al., "Planets and Debris Disks: Results from a Spitzer/MIPS Search for Infrared Excess," ApJ 705, 1226 (2009).

26. Plavchan, P., Werner, M. W., Chen, C. H., et al., "New Debris Disks Around Young, Low-Mass Stars Discovered with the Spitzer Space Telescope," ApJ 698, 1068 (2009).

27. Koerner, D. W., Kim, S., Trilling, D. E., et al., "New Debris Disk Candidates Around 49 Nearby Stars," ApJL 710, L26 (2010).

28. Eiroa, C., Fedele, D., Maldonado, J., et al., "Cold DUst around NEarby Stars (DUNES). First results. A resolved exo-Kuiper belt around the solar-iike star $\zeta^{2}$ Ret," $A \& A$ 518, L131 (2010).

29. Crepp, J. R., Pueyo, L., Brenner, D., et al., "Speckle Suppression with the Project 1640 Integral Field Spectrograph," ApJ 729, 132 (2011).

30. Beuzit, J.-L. and Feldt, M. and Dohlen, K., et al., "SPHERE: a planet finder instrument for the VLT," Proc. SPIE 7014, 41 (2008).

31. Fortney, J. J., Marley, M. S., Saumon, D., and Lodders, K., "Synthetic Spectra and Colors of Young Giant Planet Atmospheres: Effects of Initial Conditions and Atmospheric Metallicity," ApJ 683, 1104 (2008).

32. Mawet, D., Pueyo, L., Moody, D., Krist, J., \& Serabyn, E. "The Vector Vortex Coronagraph: sensitivity to central obscuration, low-order aberrations, chromaticism, and polarization Proc. SPIE 7339, 33 (2010).

33. Serabyn, E., Mawet, D., and Burruss, R., "An image of an exoplanet separated by two diffraction beamwidths from a star," Nature 464, 1018 (2010).

34. DeWeese, K. and Ward, P., "Demonstration of a Balloon Borne Arc-second Pointer Design," COSPAR Scientific Assembly 36, 2531 (2006).

35. Trauger, J., Stapelfeldt, K., Traub, W., et al., "ACCESS: a concept study for the direct imaging and spectroscopy of exoplanetary systems," Proc. SPIE 7731 (2010).

36. Trauger, J., Stapelfeldt, K., Traub, W., et al., "ACCESS: a NASA mission concept study of an Actively Corrected Coronagraph for Exoplanet System Studies," Proc. SPIE 7010 (2008).

37. Bayard, D. S., Kang, B. H., Brugarolas, P. B., and Boussalis, D., "An integrated optimal estimation approach to Spitzer Space Telescope focal plane survey," Proc. SPIE 5487, 146 (2004).

38. Brugarolas, P., Alexander, J., et al., "ACCESS pointing control system," Proc. SPIE 7731 (2010).

39. Traub, W. A., Chance, K. V., and Coyle, L. M., "Performance of a single-axis platform for balloon-borne remote sensing," Review of Scientific Instruments 57, 2519 (1986).

40. Lanzi, R.J., "Wallops Arc-Second Pointer Test Flight, Quick-Look Summary," NASA Wallops Flight Facility, internal document (2011). 
41. Traub, W. A. and Oppenheimer, B. R., "Direct Imaging of Exoplanets," in Exoplanets, The University of Arizona Press, Tucson, 111 (2010).

42. Trauger, J. T. and Traub, W. A., "A laboratory demonstration of the capability to image an Earth-like extrasolar planet," Nature 446, 771 (2007).

43. Frazier, B. W., Cavaco, J., Smith, M., and Chaudhry, Z., "Design and operation of an integrated wavefront corrector (IWC)," Proc. SPIE 5169, 90 (2003).

44. DuVarney, R., Bleau, C., Motter, G., et al., "SciMeasure Wavefront Sensor Cameras and their Application in the Palomar Adaptive Optics System," Experimental Astronomy 11, 237 (2001).

45. Krist, J. E. and Burrows, C. J., "Phase-retrieval analysis of pre-and post-repair Hubble Space Telescope images," Applied Optics 34, 4951 (1995).

46. Bordé, P. J. and Traub, W. A., "High-Contrast Imaging from Space: Speckle Nulling in a Low-Aberration Regime," ApJ 638, 488 (2006).

47. Give'on, A., Kern, B., Shaklan, S., et al., "Broadband wavefront correction algorithm for high-contrast imaging systems," 6691 (2007).

48. Heap, S. R., Lindler, D. J., Lanz, T. M., et al., "Space Telescope Imaging Spectrograph Coronagraphic Observations of $\beta$ Pictoris," ApJ 539, 435 (2000).

49. Krist, J. E., Stapeifeldt, K. R., Bryden, G., et al., "HST and Spitzer Observations of the HD $207: 29$ Debris Ring," AJ 140, 1051 (2010).

50. Lafrenière, D., Marois, C., Doyon, R., et al., "A New- Algorithm for Point-Spread Function Subtraction in High-Contrast Imaging: A Demonstration with Angular Differential Imaging," ApJ 660, 770 (2007).

51. Roberge, A. et al., "The Exozodiacal Dust Problem for DIrect Observation of ExoEarths," PASP, in press, (2012). 